\title{
Study on Multithreshold Segmentation Method for Color Image Zheng Wen-kui*1a ${ }^{1 \mathrm{~L}}$ Liu kun ${ }^{1 \mathrm{~b}}$ \\ 1. School of Software, Henan University, Kaifeng 475004, China zhengwenkuisja@163.com
}

Keywords: Color image; Multithreshold; Segmentation method

\begin{abstract}
With the development of science and technology, the application of color image is becoming increasingly wide. The traditional image processing techniques has been widely recognized by scholars from all over the world. The traditional color image segmentation technology runs a simple classified processing in pixels and splits with a single scale. After image segmentation, the overall performance is not good, and the vision is blurred. For this reason, this paper proposed a multithreshold segmentation method for color image, which sets the doublet segmentation parameters by threshold histogram and improves the automatic choice of threshold value and image fusion. The simulation results show that this method is more flexible in the process of color image segmentation. The accuracy of the image is higher. The fuzzy effect after original image segmentation has been changed. It has great application value.
\end{abstract}

\section{Introduction}

Color image segmentation techniques is always a classic image processing problem. When stepping into the digital era, it has become a focus question of image segmentation. The clarity of color image threshold segmentation and accuracy of data will directly affect the effectiveness of image post-processing.

In the process of segmentation and data extraction for target area, the traditional color image segmentation method is mainly based on the single pixel in color image for the processing of the gray scale, the color and the texture. The target region image of color image corresponds to many similar single pixels. The fusion effect after data extraction and image segmentation is bad. The image after segmented is fuzzy. This is harmful to the progress in later task.

Therefor this paper proposed a multithreshold segmentation method for color image, which sets the doublet segmentation parameters by threshold histogram and improves the automatic choice of threshold value and image fusion. The simulation results show that this method is more flexible in the process of color image segmentation. The accuracy of the image is higher. The fuzzy effect after original image segmentation has been changed. It is helpful for the later development of the task. It has great value in practical application.

\section{Threshold segmentation principle}

The image threshold segmentation divides the whole image data into target area and background area by using the target area pixels to be extracted in the image and the grayscale difference of all the target pixels set in the whole image. It selects a reasonable threshold parameter to determine the point target of each pixel and produces the corresponding binary image.

The main feature of threshold segmentation is that it has strong analysis ability to correlation between the pixels of the similar image, which can make a difference. It can find the boundaries of the segmented regions in the images.

Setting the original color image region as $\mathrm{f}(\mathrm{x}, \mathrm{y})$, with the certain rules, we can find the appropriate segmentation parameter gray value in $\mathrm{f}(\mathrm{x}, \mathrm{y})$, and determine the segmentation threshold $\mathrm{T}$, thus the color image $\mathrm{g}(\mathrm{x}, \mathrm{y})$ after segmentation is expressed in formula: 


$$
g(x, y)=\left\{\begin{array}{l}
1, f(x, u) \geq t \\
0, f(x, y) \prec t
\end{array}\right.
$$

The color image threshold is set in a range. $[T, t]$, the color image pixels in the gray level range are set to 1 , the others are set to 0 :

$$
g(x, y)=\left\{\begin{array}{l}
1, t_{2} \leq(x, u) \geq t_{1} \\
0, \text { other }
\end{array}\right.
$$

In a particular situation, pixels above its threshold can keep the original color image data. The other pixels become zero. This segmentation method is called color image threshold segmentation method, color image pixel after segmentation is:

$$
g(x, y)=\left\{\begin{array}{l}
f(x, y),(x, u) \geq t_{1} \\
0, \text { other }
\end{array}\right.
$$

The color image graying segmentation pixels are shown in Figure 1.

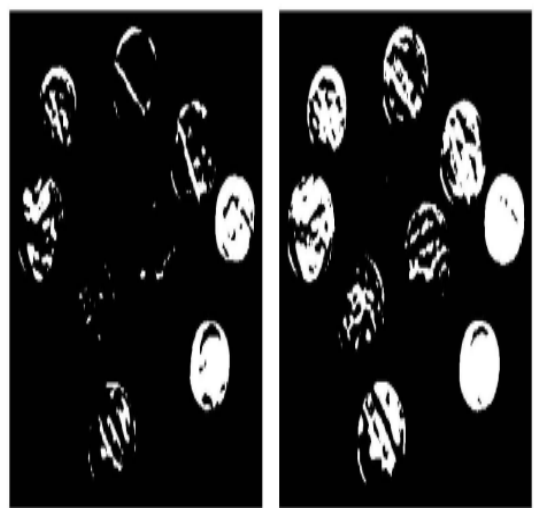

Figure 1. Diagrammatic sketch of threshold segmentation

\section{Color image multithreshold segmentation method}

\section{A. Multithreshold segmentation}

The threshold is used to segment the image. The multithreshold image segmentation is established. Rebuilding with the multidimensional corridor chart, it is split by the minimum robust error. Its specific multithreshold model is shown in Figure 2.

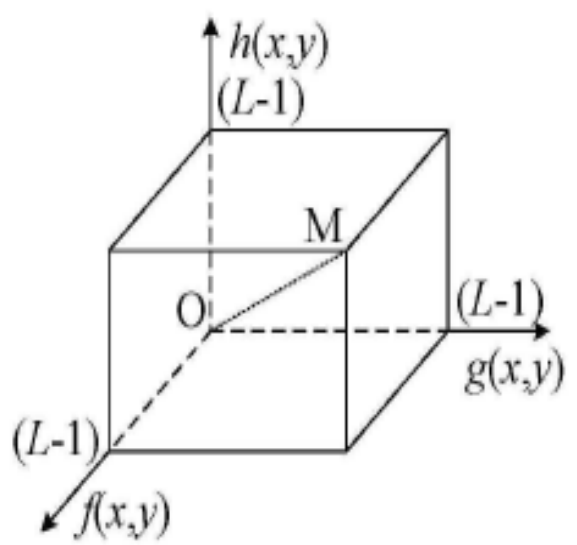

Figure 2. Multithreshold image segmentation model 
Ideally, the pixel data of the point $(\mathrm{x}, \mathrm{y})$ in the multithreshold interference of the color image is $\mathrm{f}$ $(\mathrm{x}, \mathrm{y})$. Its average parameters are similar to the modeling. It is in formulas:

$$
\cos \alpha=\frac{|O A|}{|O M|}=\frac{|O A|}{\sqrt{3}|O A|}=\frac{\sqrt{3}}{3}
$$

The multithreshold is expressed as a formula after reduced the pixel projection value of the color image:

$$
|O N|=|O B| \cos \alpha=\frac{\sqrt{3}}{3}(f+g+h)
$$

Table 1 intuitively shows the complexity of multithreshold segmentation method

Table 1 Time complexity of multithreshold segmentation

\begin{tabular}{cc}
\hline Segmentation method & Time complexity \\
\hline One-dimensional minimum error algorithm & O(L) \\
Two-dimensional minimum error algorithm & O(L) \\
$\begin{array}{c}\text { Multidimensional value threshold } \\
\text { segmentation algorithm }\end{array}$ & O(L) \\
\hline
\end{tabular}

\section{B. Histogram two-peak method}

In the actual application state, the histogram two-peak method has large difference on background and the extracting of the gray values of color region, which is reflected in the gray histogram of color image. This is the obvious bimodal distribution of the histogram. The pixel is judged by gray level and threshold value. The splitted threshold parameter is analyzed to find the image threshold t. It is shown in Figure 3.

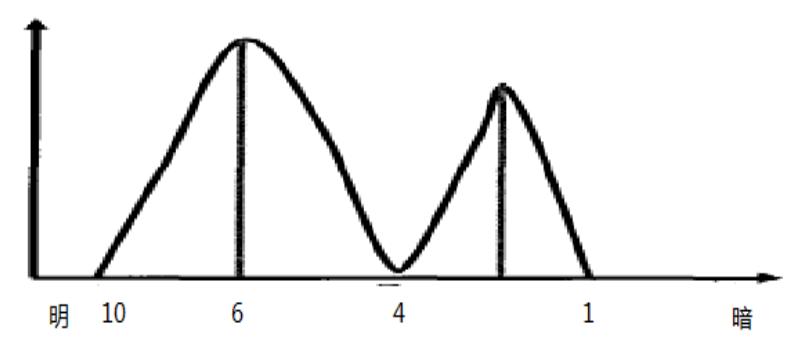

Figure 3. Multithreshold histogram

\section{Simulation experiment}

In order to test the effectiveness of the multithreshold segmentation method of color image, we ran the MATLAB simulation experiment based on color image segmentation. A variety of color image segmentation methods were tested. Its data statistics table is shown in Table 2.

\begin{tabular}{llllll}
\hline $\begin{array}{l}\text { Test pattern } \\
\text { number }\end{array}$ & $\begin{array}{l}\text { Initial } \\
\text { threshold }\end{array}$ & $\begin{array}{l}\text { Optimal } \\
\text { threshold }\end{array}$ & $\begin{array}{l}\text { Optimal } \\
\text { variance }\end{array}$ & Iterations & $\begin{array}{l}\text { Iterative } \\
\text { variance }\end{array}$ \\
\hline $\mathrm{A}$ & $\mathrm{T}=0.5 \mathrm{~L}$ & 122.1 & 18.7 & 33.2 & 335.3 \\
$\mathrm{~B}$ & $\mathrm{~T}=0.5 \mathrm{~L}$ & 121.1 & 8.7 & 12.2 & 17.1 \\
$\mathrm{C}$ & $\mathrm{T}=0.5 \mathrm{~L}$ & 123.4 & 12.8 & 9.7 & 32.1 \\
$\mathrm{D}$ & $\mathrm{T}=0.5 \mathrm{~L}$ & 114.2 & 28.3 & 11.1 & 323.3 \\
$\mathrm{E}$ & $\mathrm{T}=0.5 \mathrm{~L}$ & 123.7 & 78.9 & 22.1 & 58.9 \\
$\mathrm{~F}$ & $\mathrm{~T}=0.5 \mathrm{~L}$ & 78.9 & 23.3 & 44.9 & 321.9 \\
$\mathrm{G}$ & $\mathrm{T}=0.5 \mathrm{~L}$ & 99.3 & 22.0 & 64.9 & 88.6 \\
\hline
\end{tabular}

The color image information in the simulation experiment was used to segment the data, and decomposed and extracted the pixels of the color image. We did the segmentation speed comparison 
with the traditional image segmentation method and the multithreshold doublet segmentation method proposed in this paper. The results was shown in Figure 4.

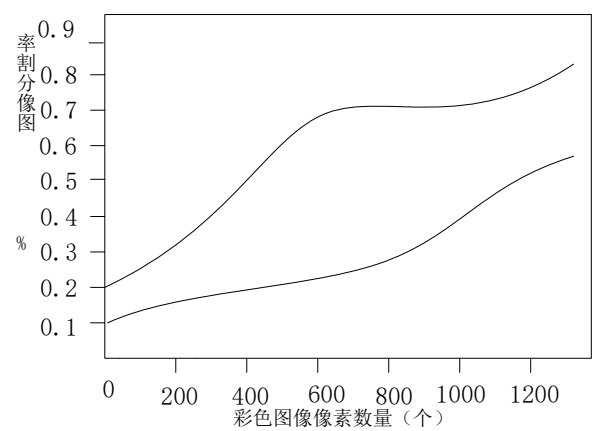

Figure 4. Comparison chart of color image segmentation speed in two methods

In color image segmentation, the segmentation speed of the multithreshold histogram doublet segmentation method was faster. The segmentation stability was obviously higher than the traditional method. The adaptive ability was strong.

The color image pixel data was known. The comparison of the traditional image segmentation algorithm and the proposed method in dealing with fault tolerance of color image pixels was shown in Figure 4:

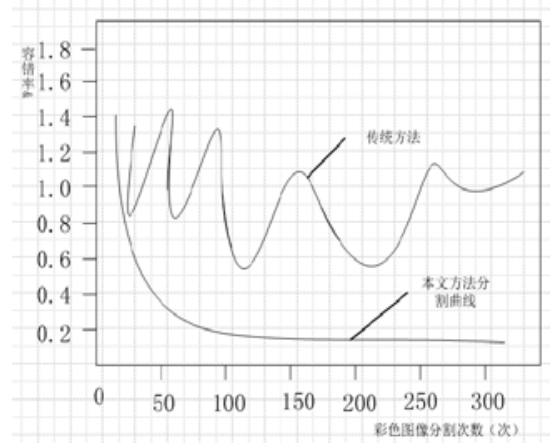

Figure. 5 Comparison chart of fault tolerance in the two methods

As shown in Figure 5, in the processing of the color image segmentation, the speed of histogram doublet image segmentation based on multithreshold is faster. The fault tolerance was higher. The clarity of the image was higher.

From the experimental data, using the the multithreshold histogram doublet image segmentation to segment the color image is faster. The fault-tolerant rate is increasing. The degree of fuzzy is reduced. We effectively understand the defects of the traditional method.

\section{Conclusions}

In the process of segmentation and data extraction for target area, the traditional color image segmentation method is mainly based on the single pixel in color image for the processing of the gray scale, the color and the texture. The target region image of color image corresponds to many similar single pixels. The fusion effect after data extraction and image segmentation is bad. A multithreshold segmentation method for color image was proposed in this paper, which set the doublet segmentation parameters by threshold histogram and improved the automatic choice of threshold value and image fusion. The simulation results show that this method is more flexible in the process of color image segmentation. The accuracy of the image is higher. The fuzzy effect after original image segmentation has been changed. It has great application value.

\section{References}

[1] Felzenszwalb P F, Huttenlocher D P. Efficient Graph-Based Image Segmentation[J]. International Journal of Computer Vision, 2004, 59(2):167-181.

[2] Grady L. Random Walks for Image Segmentation[J]. IEEE Transactions on Pattern Analysis \& 
Machine Intelligence, 2006, 28(11):1768-1783.

[3] Noble J A, Boukerroui D. Ultrasound image segmentation: a survey[J]. IEEE Transactions on Medical Imaging, 2006, 25(8):987.

[4] Pham D L, Xu C, Prince J L. Current Methods in Medical Image Segmentation1[J]. Annual Review of Biomedical Engineering, 2000, 2(2):315.

[5] Pal N R, Pal S K. A Review on Image Segmentation Techniques[J]. Pattern Recognition, 1993, 26(9):1277-1294.

[6] Vese L A, Chan T F. A Multiphase Level Set Framework for Image Segmentation Using the Mumford and Shah Model[J]. International Journal of Computer Vision, 2002, 50(3):271-293.

[7] Boykov Y, Funka-Lea G. Graph Cuts and Efficient N-D Image Segmentation[J]. International Journal of Computer Vision, 2006, 70(2):109-131.

[8] Malik J, Belongie S, Leung T, et al. Contour and Texture Analysis for Image Segmentation[J]. International Journal of Computer Vision, 2001, 43(1):7-27.

\section{Author introduction}

Correspondence Author:Zheng Wenkui (1982-), male (Han), Henan, Zhengzhou, master's degree, lecturer, the main research areas for algorithm analysis and network security;

Liu Kun (1997-) male (Han), Henan Luoyang, the main research direction: Software Engineering. 